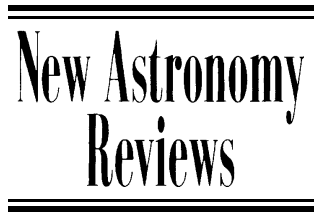

www.elsevier.com/locate/newastrev

\title{
QUEST on DASI: a South Pole CMB polarization experiment
}

Sarah Church ${ }^{\mathrm{a}, *}$, Peter Ade ${ }^{\mathrm{b}}$, James Bock ${ }^{\mathrm{c}}$, Melanie Bowden ${ }^{\mathrm{b}}$, John Carlstrom ${ }^{\mathrm{d}}$, Ken Ganga ${ }^{\mathrm{e}}$, Walter Gear ${ }^{\mathrm{b}}$, James Hinderks ${ }^{\mathrm{a}}$, Wayne $\mathrm{Hu}^{\mathrm{d}}$, Brian Keating ${ }^{\mathrm{f}}$, John Kovac ${ }^{\mathrm{d}}$, Andrew Lange ${ }^{\mathrm{f}}$, Eric Leitch ${ }^{\mathrm{d}}$, Olivier Mallie ${ }^{\mathrm{b}}$, Simon Melhuish ${ }^{\mathrm{b}}$, Anthony Murphy ${ }^{\mathrm{g}}$, Ben Rusholme ${ }^{\mathrm{a}}$, Creidhe O'Sullivan ${ }^{\mathrm{g}}$, Lucio Piccirillo ${ }^{\mathrm{b}}$, Clem Pryke ${ }^{\mathrm{d}}$, Andy Taylor ${ }^{\mathrm{h}}$, Keith Thompson ${ }^{\mathrm{a}}$

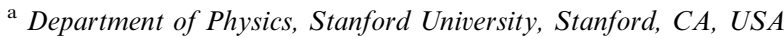

${ }^{\mathrm{b}}$ Department of Physics and Astronomy, University of Wales, Cardiff, UK

${ }^{\mathrm{c}}$ Jet Propulsion Laboratory, Pasadena, CA, USA

d Department of Physics, University of Chicago, Chicago, IL, USA

${ }^{\mathrm{e}}$ Infrared Processing and Analysis Center, Caltech, Pasadena, CA, USA

${ }^{\mathrm{f}}$ Department of Physics, Maths and Astronomy, Caltech, Pasadena, CA, USA

${ }^{g}$ Department of Experimental Physics, National University of Ireland, Maynooth, Ireland

${ }^{\mathrm{h}}$ Institute for Astronomy, University of Edinburgh, Edinburgh, UK

\begin{abstract}
QUEST on DASI is a ground-based, high-sensitivity, high-resolution $\left(\ell_{\max } \sim 2500\right)$ experiment designed to map $\mathrm{CMB}$ polarization at 100 and $150 \mathrm{GHz}$ and to measure the power spectra from $E$-modes, $B$-modes from lensing of the $\mathrm{CMB}$, and $B$-modes from primordial gravitational waves. The experiment comprises a $2.6 \mathrm{~m}$ Cassegrain optical system, equipped with an array of 62 polarization-sensitive bolometers (PSBs), located at the South Pole. The instrument is designed to minimize systematic effects; features include differencing of pairs of orthogonal PSBs within a single feed, a rotatable achromatic waveplate, and axisymmetric rotatable optics. In addition the South Pole location allows both repeatable and highly controlled observations. QUEST on DASI will commence operation in early 2005.
\end{abstract}

(C) 2003 Elsevier B.V. All rights reserved.

\section{The QUEST on DASI experiment}

We are building a ground-based polarization experiment called QUEST ( $Q U$ Extra-galactic Survey Telescope) on DASI (the Degree Angular Scale Interferometer). In late 2004, the existing

\footnotetext{
${ }^{*}$ Corresponding author.

E-mail address: schurch@stanford.edu (S. Church).
}

DASI receivers and feedhorns will be removed and replaced with a bolometric receiver mounted on a $2.6 \mathrm{~m}$ Cassegrain telescope. First light is expected in early 2005. QUEST on DASI is intended not only to provide detailed measurements of the $E$ mode power spectrum, but also to measure the $B$-mode power spectrum from the gravitational lensing of the CMB by intervening matter. Also, depending on the magnitude of the tensor-scalar ratio, QUEST on DASI could detect, or set strong 
limits to the amplitude of, the $B$-mode power spectrum from primordial gravity waves.

Since the CMB polarization signal is an order of magnitude fainter than the temperature anisotropy signal, instruments designed for CMB polarization measurements must have both a significant increase in raw sensitivity over temperature experiments and a comparable improvement in the control of systematic errors. In this paper we discuss the design of QUEST on DASI with particular emphasis on the goal of minimizing systematics.

\section{Instrument specifications and design philosophy}

The specifications of QUEST on DASI are listed in Tables 1 and 2. They include a large field of view and a large number of pixels to achieve the high instantaneous sensitivity required to permit fast mapping of large areas of sky (the instanta-

Table 1

The specifications of QUEST on DASI

\begin{tabular}{lcc}
\hline Telescope & & \\
Size of primary mirror $(\mathrm{m})$ & 2.64 & \\
Total field of view $\left(^{\circ}\right)$ & 1.5 & \\
Pointing accuracy (arcmin, & 0.1 & \\
$\quad$ reconstructed) & & \\
Receiver & & \\
Frequency band (GHz) & 100 & 150 \\
Number of bolometers (two per feed) & 24 & 38 \\
Angular resolution (arcmin) & 6.3 & 4.2 \\
Bandwidth (\%) & 25 & 25 \\
End-to-end optical efficiency & 0.45 & 0.45 \\
System temperature inc. atmosphere $(\mathrm{K})$ & 24 & 24 \\
NET per bolometer $\left(\mu \mathrm{K} \mathrm{s}\right.$ s $^{-1 / 2}$ ) & 270 & 300 \\
\hline
\end{tabular}

The optical efficiency, system temperature and NET are expected values based on similar experimental designs.

Table 2

Expected noise performance of each PSB, showing the contributions from photon noise (Phot.), bolometer noise (Bol., including amplifier noise of $12 n \mathrm{~V} \mathrm{~Hz}^{-1 / 2}$ )

\begin{tabular}{lllll}
\hline $\begin{array}{l}\text { Freq. } \\
(\mathrm{GHz})\end{array}$ & $\begin{array}{l}\text { Bandwidth } \\
(\Delta v / v)\end{array}$ & \multicolumn{4}{l}{ NET $\left(\mu \mathrm{K} \mathrm{s}^{-1 / 2}\right)$} \\
\cline { 3 - 5 } & & Phot. & Bol. & Total \\
\hline 100 & 0.25 & 204 & 177 & 270 \\
150 & 0.25 & 244 & 175 & 300 \\
\hline
\end{tabular}

The end-to-end performance of both the cold and warm prototype readout electronics has been verified. neous sensitivity at $150 \mathrm{GHz}$ is similar to that expected for the Planck satellite). The angular resolution of the experiment has been chosen to give a cutoff in $\ell$-space of about 2500 , in order to span the expected peak in both the $E$-mode signal, and the $B$-mode signal from gravitational lensing. The minimum $\ell$ that can be mapped by a singledish experiment such as QUEST on DASI is set by the scan length and by stability limitations that depend on both electronic and atmospheric noise. For QUEST on DASI, we expect the minimum to be somewhere between $\ell=25$ and 100 .

Raw sensitivity is insufficient, by itself, to define the ultimate performance of an experiment - systematic effects must also be reduced to equally low levels. QUEST on DASI is designed first to minimize, and second to characterize, residual systematics. Simultaneous observations at 100 and $150 \mathrm{GHz}$ will allow spectral discrimation of true signal from foregrounds (see Section 3). Many layers of differencing will be used to minimize systematic contamination to the data in the signal band. The most important is the differencing of two polarization-sensitive bolometers (PSBs) located within the same feedhorn. The output after differencing the two PSBs is

$$
\Delta I \propto Q \cos 2 \theta+U \sin 2 \theta,
$$

where $Q$ and $U$ are the Stokes parameters of interest and $\theta$ is the angle between the instrument coordinate system and the sky coordinate system. These detectors were developed for Planck and were recently flown on the second BOOMERANG flight (Jones et al., 2002; Montroy et al., 2003). This differential detector system rejects commonmode noise fluctuations in the incoming signal, in particular, unpolarized fluctuations in atmospheric emission. The instantaneous rejection afforded by locating both of the detectors in the same feedhorn is much greater than that from spatial differencing methods such as chopping.

The residual common-mode signal after differencing two PSBs is dominated by the effects of instrumental polarization which converts a fraction of the unpolarized emission from the atmosphere, telescope and CMB to a polarized signal. We can distinguish true sky polarization from instrumen- 
tally polarized signals by rotating the instrument with respect to the sky. In QUEST on DASI we have two means of accomplishing this - first we can rotate the entire instrument (telescope plus receiver, see Section 2.1) about the optic axis and second we have a half-waveplate (Section 2.2) which can rotate the coordinate system of each PSB pair and feedhorn with respect to both the telescope and the sky. These two degrees of freedom will allow us to fully characterize all sources of instrumental polarization, and to clean residual common-mode signals from the data.

\subsection{Optical design}

Fig. 1 shows a schematic of the QUEST on DASI system. The Cassegrain design and associated reimaging optics are shown in Fig. 2. Two 18-cm-diameter lenses, constructed from antireflection-coated silicon, are used to match the slow beam from the secondary to the F2.3 focal surface. The re-imaging optics permit a large field of view, while keeping the curvature of the focal plane, the size of the feedhorns, and the size and curvature of the lenses within manufacturable limits. A Casse-
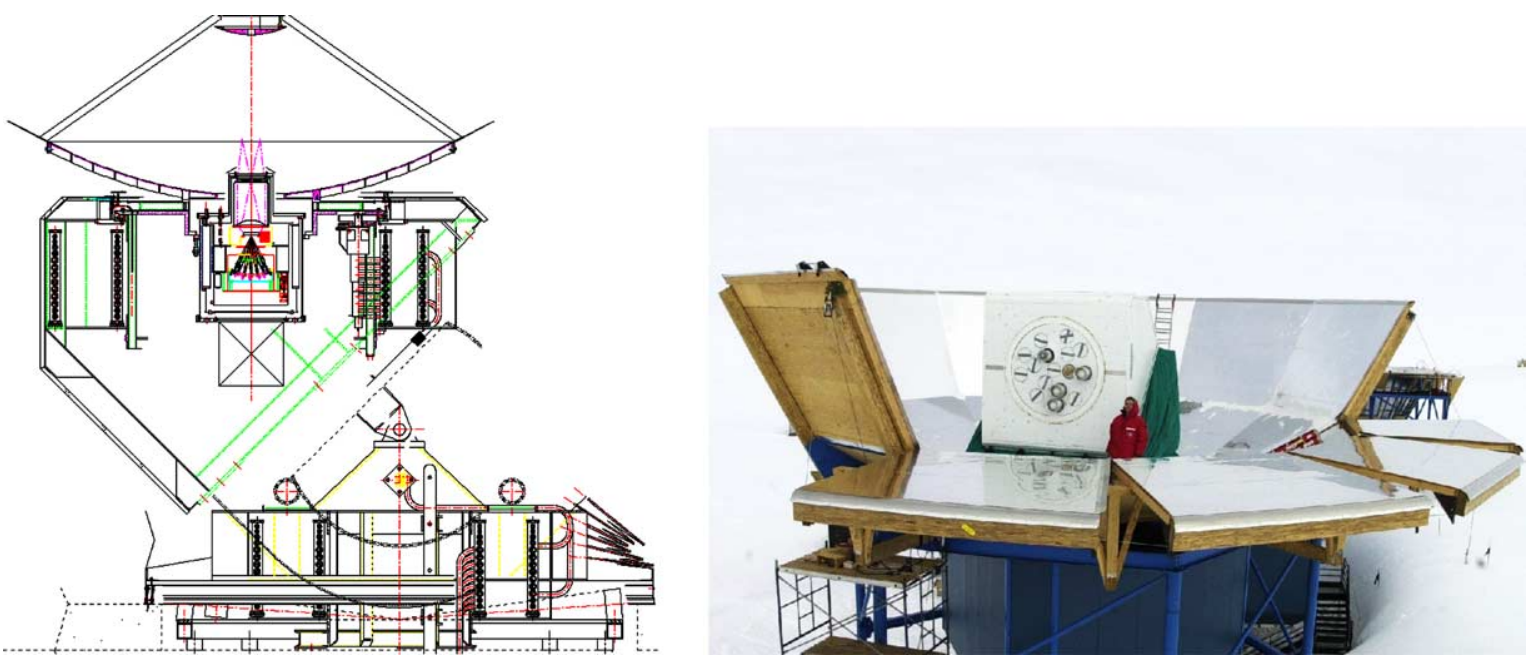

Fig. 1. Left: Schematic showing how the QUEST telescope will attach to the DASI mount. The mount is alt-az, and permits rotation of the telescope and receiver about the optic axis. The receiver is housed inside an enclosed structure to permit easy access to the instrument during the Polar winter. Right: DASI with its ground-shield lowered.
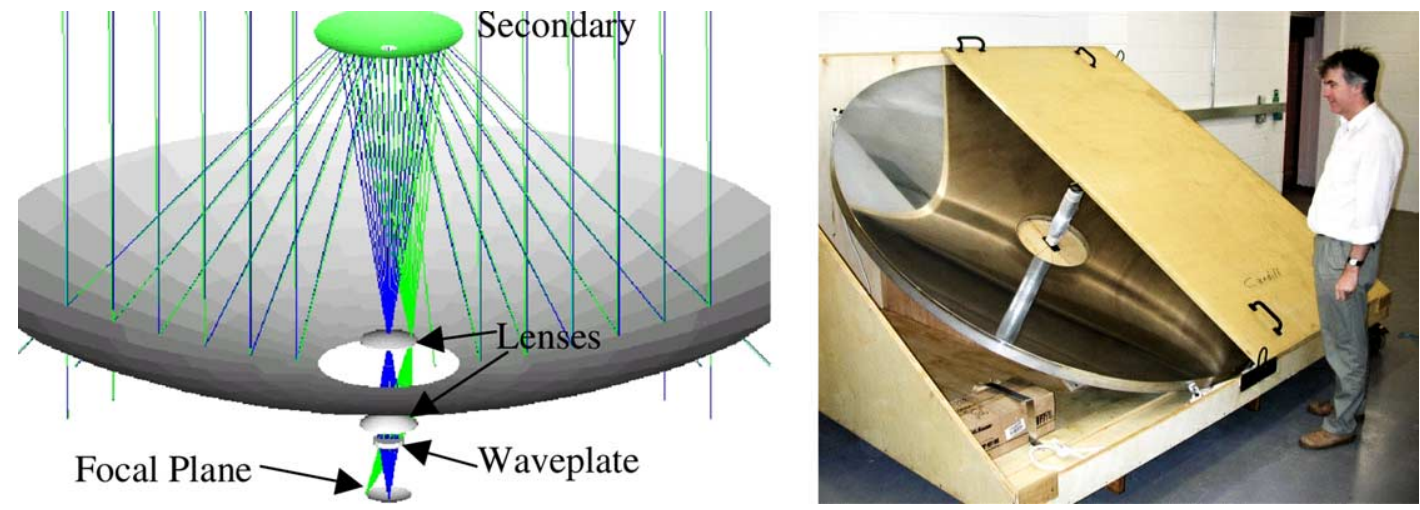

Fig. 2. Left: Layout of the QUEST optical system. Right: Prof. Walter Gear with the QUEST primary mirror after delivery from the manufacturer, Marcon (Italy). 
grain design was chosen for its ease of construction, and because of its symmetry, enabling the polarization properties of the system to be quantified and removed by rotating the entire instrument about the optic axis. This rotational degree of freedom is already built into the DASI mount (Fig. 1).

Cassegrain designs have been used before in CMB polarization experiments such as COMPASS (Farese et al., 2003) and their optimization for low sidelobes/spillover is well understood. QUEST on DASI incorporates the following features:

- The primary is under-illuminated by corrugated conical feedhorns (Section 2.3) to minimize the effects of ground spillover.

- A cold Lyot stop is incorporated to truncate offaxis rays.

- The secondary is supported with a continuous cone of RF transparent foam rather than discrete supports which would break the axial symmetry (this technique was used in COMPASS, Farese et al., 2003).

- A small aperture in the center of the secondary prevents the detectors from seeing warm cryostat emission (this hole will also be used to inject a signal of known polarization directly into the optical system for regular gain calibration). Additional baffling around the cryostat window will further ensure that the detectors see only the sky. In order to maintain high sensitivity, it is essential to minimize warm loading from the optical components. All dielectric components - the foam cone, cryostat window, lenses and waveplate (see next section) - are manufactured from low-loss materials. The lenses and waveplate are anti-reflection coated and cooled to $4 \mathrm{~K}$. The expected contributions to the loading are shown in Table 3.

\subsection{Waveplate}

An achromatic rotating half-waveplate is located close to the pupil stop of the system (see Fig. 2). The function of this waveplate is to enable the rotation of the coordinate system of the detectors with respect to the sky without moving the detectors themselves on the sky to enable the measurement of both $Q$ and $U$ by each PSB pair. Because of microphonic considerations, we do not
Table 3

Expected contributions to optical loading at $150 \mathrm{GHz}$

\begin{tabular}{lc}
\hline Component & $T_{\text {load }}(\mathrm{K})$ \\
\hline Atmosphere & 10.0 \\
Telescope & 8.2 \\
Window & 4.0 \\
Lenses, waveplate and filters & 1.2 \\
CMB & 0.6 \\
Total & 24.0 \\
\hline
\end{tabular}

intend to continuously rotate the waveplate. Instead the telescope will be scanned in azimuth for 1-2 min (Section 3), the waveplate rotated to change the orientation of the PSB coordinate system with respect to the sky, and the scan repeated.

The waveplate itself is constructed from five layers of anti-reflection coated birefringent sapphire. The design is predicted to achieve broadband spectral transmission better than $98 \%$, with cross-polarization under $0.5 \%$. We are using sapphire rather than lower birefringence quartz as this reduces the thickness of the assembly to just 15 $\mathrm{mm}$. By locating the waveplate near an image of the primary mirror, its diameter is minimized. Also all detectors view the entire waveplate, minimizing systematic changes in loading or instrumental polarization as the plate is rotated.

The waveplate rotation mechanism is also located at $4 \mathrm{~K}$ to simplify the design of the cryostat. The rotation will be driven by a stepper motor (Berger-Lahr VRM564). The motor is modified for cryogenic use by replacing the supplied bearings with cryogenic bearings that incorporate relaxed mechanical tolerances to prevent seizure on contraction, and dry lubrication (Dow Corning 321). The motor is currently undergoing extensive lifetime testing at cryogenic temperatures and has been found to work reliably and with low power consumption $(\sim 100 \mathrm{~mW})$. The rest of the waveplate mechanism will comprise an angular contact bearing and a Cryomagnetics Hall sensor to encode the waveplate position.

\subsection{The focal plane}

The QUEST on DASI focal plane will contain 31 feeds and associated PSBs, 19 at $150 \mathrm{GHz}$ and 
12 at $100 \mathrm{GHz}$, as per Table 1 . The corrugated, profiled feeds have been designed to provide a well-controlled beam, low sidelobes and low crosspolarization. The horns have been manufactured by TK Ltd. (UK) and are currently undergoing optical testing.

The focal plane assembly is shown in Fig. 3. The feedhorns are located on the spherical focal surface in a hexagonal close-packed array designed for optimum redundancy, filling factor and frequency overlap. The PSBs, at the back of the feedhorns, are orientated so that both Stokes parameters are sampled by different feeds during azimuthal scans of the telescope. At the end of a scan, the wave plate is rotated, and the scan repeated, allowing a cross-check of the $Q$ and $U$ signals measured by different feeds.

\subsection{Observing frequencies and foregrounds}

QUEST will simultaneously observe in two bands centered close to 100 and $150 \mathrm{GHz}$ that span atmospheric windows of high-transparency. Simultaneous multi-frequency observations are im- portant for proper rejection of any atmospheric residuals and any variable (synchrotron) sources in the data. The frequency bands are defined by the high-pass waveguide cutoff of the horn throat and by a metal-mesh low-pass edge located at the entrance aperture of the horn. This filtering scheme has been extensively used by CMB experiments including Boomerang (Montroy et al., 2003), ACBAR (Runyan et al., 2003) and Archeops (Benoit et al., 2003).

Atmospheric noise is a concern for all groundbased experiments, including polarization experiments. The South Pole site lies at an effective altitude of $3200 \mathrm{~m}$ due to the lower scale height of the atmosphere at the pole and is extremely dry due to the freezing out of most of the water vapor, leading to very high transparency. Both atmospheric studies (Lay and Halverson, 2000; Peterson et al., 2003) and successful ground-based bolometer experiments (Runyan et al., 2003) have shown exceptional atmospheric stability at the Pole. This will reduce both the error in the reconstruction of the data and the lowest $\ell$ that can be sampled by a fixed scan rate on the sky.

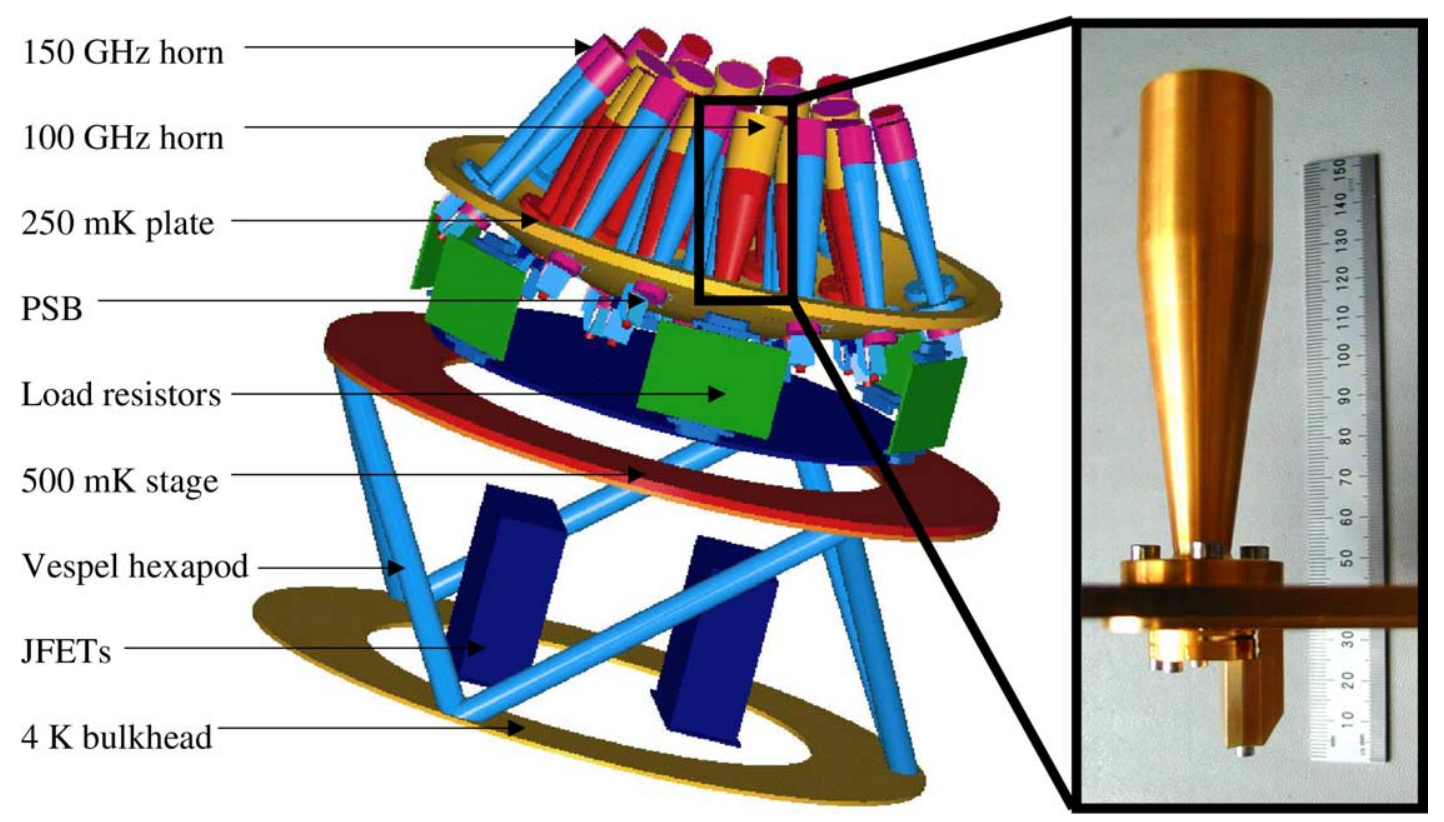

Fig. 3. Left: CAD model of the QUEST on DASI focal plane assembly, with shielding removed. Right: Photograph of a $100 \mathrm{GHz}$ horn and PSB module assembled for testing. 

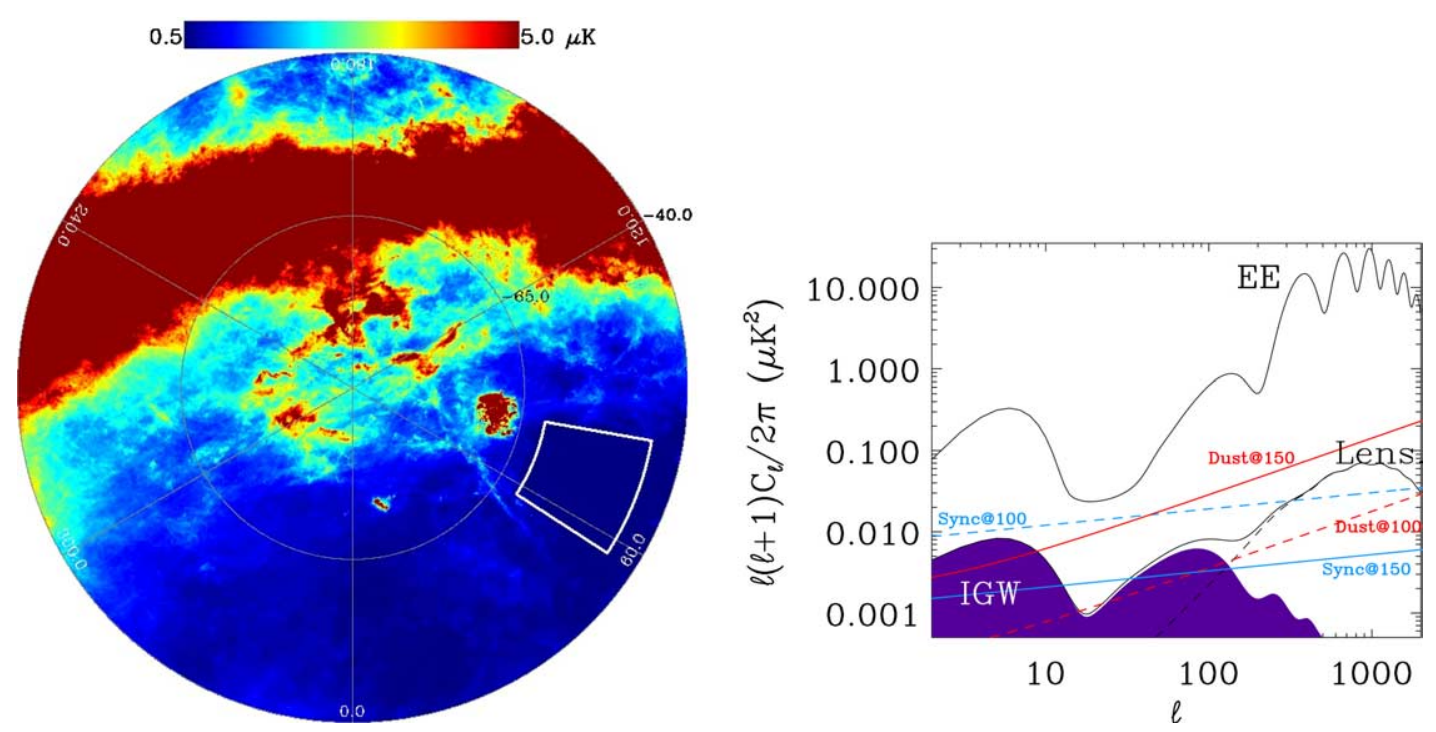

Fig. 4. Equal area zenithal projection showing foreground levels in sky that is accessible to QUEST on DASI. The Southern Celestial Pole is located in the center of the plot, declination $-45^{\circ}$ is the perimeter, and RA is labelled around the edge. A possible 300 deg ${ }^{2}$ survey region is bordered in white. Right: foreground estimates as a function of angular scale. The two solid lines, red and blue, correspond to dust and synchrotron contaminants at $150 \mathrm{GHz}$, estimated from Prunet et al. (1998) and Baccigalupi et al. (2001), respectively. The dashed lines are the estimates at $100 \mathrm{GHz}$.

The sky above the South Pole features large areas clear of foregrounds such as dust and synchrotron radiation (see Fig. 4). These projections have been confirmed to $\sim 10 \mu \mathrm{K}$ levels by other $\mathrm{CMB}$ experiments and recent measurements from WMAP (Bennett et al., 2003). Fig. 4 shows a 300 $\mathrm{deg}^{2}$ region of sky that is a possible target for observation by QUEST on DASI. The right hand side of the figure shows conservative estimates of the foreground contamination as a function of angular scale. We expect the foregrounds measured by QUEST on DASI to be somewhat lower because it is a pointed experiment, allowing us to target the lowest foreground regions.

\section{Observational strategy and predicted results}

We expect to operate QUEST on DASI for at least two years beginning in early 2005. Observations will be continuous over the polar winter. The experiment has been designed (based on the experience of DASI and re-using much of the existing control system) for both remote and automatic operation, and will only require the attention of one full-time person on site over the Polar winter.

At the South Pole the sky only moves in azimuth relative to the ground and so the same region can be observed continuously through the Polar

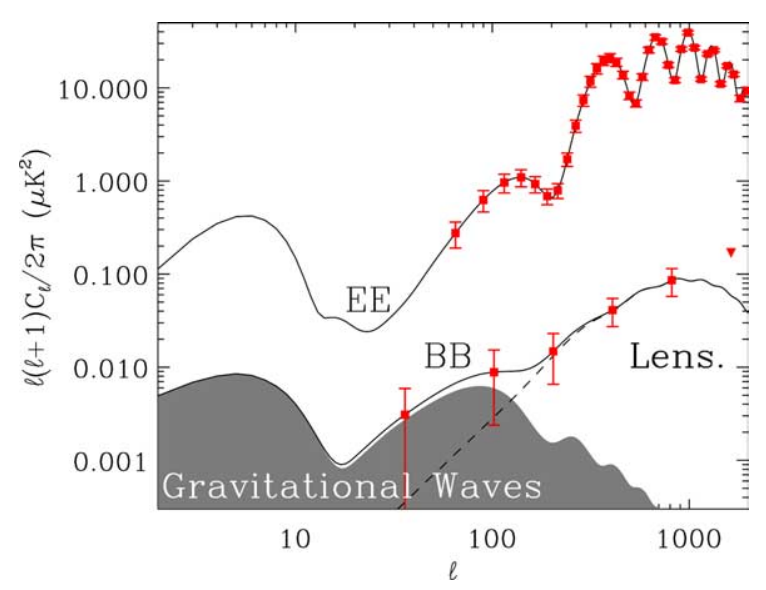

Fig. 5. The projected sensitivity of QUEST on DASI after two polar winters. A tensor scalar ratio of $r=0.1$ is assumed. The triangle is an upper limit. 
winter, and ground-spillover can be tightly controlled. QUEST on DASI observations will be built around 1-2 min scans at constant elevation to take advantage of this geometry and to avoid changes of airmass and ground spillover which can lead to time-dependent offsets in the data. We expect to observe a region of about $300 \mathrm{deg}^{2}$ which gives good sensitivity to both the $E$ - and $B$-mode power spectra. The expected power spectrum recovery from two winters of observation is shown in Fig. 5. Detailed discussion of the likely science results from QUEST on DASI can be found in Bowden et al., 2003.

\section{Acknowledgements}

This material is based upon work supported by the National Science Foundation under Grants 9987360 and 0096778, by the Particle Physics and
Astronomy Research Council in the UK, and by the Irish Research Council for Science, Engineering and Technology.

\section{References}

Bennett, C.L. et al., 2003. ApJ 583, 1.

Bowden, M., et al., 2003. MNRAS, submitted.

Benoît, A. et al., 2003. A\&A 399, L19.

Farese, P.C., et al., 2003. These Proceedings. Available from $<$ astro-ph/0305608>.

Lay, O., Halverson, N., 2000. ApJ 543, 787.

Jones, W.C., Bhatia, R.S., Bock, J.J., Lange, A.E., 2002. In: SPIE Proceedings, Waikaloa. Available from <astro-ph/ 0209132>.

Montroy, T., et al., 2003. These Proceedings. Available from $<$ astro-ph/0305593>.

Peterson, J.B., Radford, S.J.E., Ade, P.A.R., Chamberlin, R.A., O'Kelly, M.J., Peterson, K.M., Schartman, E., 2003. PASP 115, 383-388.

Runyan, M.C., et al., 2003. These Proceedings. Available from <astro-ph/0305553>. 